\title{
Cross-platform Public Opinion of Hot Event under the Media Convergence
}

\author{
Jianping Chai ${ }^{1}$ \\ College of Information Engineering, Communication University of China, Beijing, 100024,China \\ E-mail:jp_chai@sina.com
}

Beibei Zhang

College of Information Engineering, Communication University of China, Beijing, 100024,China E-mail:2436528842@qq.com

\section{Hongjun Zhou}

College of Information Engineering, Communication University of China, Beijing, 100024,China E-mail:805530045@qq.com

\section{Fulian Yin}

College of Information Engineering, Communication University of China, Beijing, 100024,China E-mail:yinfulian@cuc.edu.cn

In view of deficiency of the cross-platform public opinion research about hot event under the media convergence, the scheme of the cross-platform public opinion research about hot event was put forward. Through calculating the public opinion index on TV platform and weibo platform based on the radio and television database as well as weibo database, this paper makes single-platform and cross-platform charts to represent the attention tendency of public opinions at different stages and on different media. The experiment proves that the method shows the propagation law of public opinion and the connected effect in a reasonable way on different media.

CENet 2015

12-13 September 2015

Shanghai, China

\section{${ }^{1}$ Speaker}




\section{Introduction}

The media convergence is an emerging media phenomenon in the context of media technology revolution, which has resulted in a lot of attention of scholars both at home and abroad $^{[1,2]}$; at meanwhile, the media convergence has brought about a revolution of propagation mode. Whenever a hot events occurs, relevant messages will flow into the society in different ways and spread explosively. Different media platforms, the traditional media, represented by audio and television, and the new media, represented by Internet, act differently to the event. Both of them play an important role in the development of public opinions.

In recent years, the research of Internet public opinion has become the focus of the public opinion research. The research of the internet public opinion theory, technology and practice has made great development ${ }^{[3,4,5,6]}$. The technological researches on Internet public opinion analysis have been developed more maturely in West, including researches on network transmission of the information content, represented by the TDT (Topic Detection and Tracking) system, which can recognize and track the hot topic ${ }^{[7]}$, and public opinion network structure analysis, which focused on internal communication mechanism of public opinion ${ }^{[8]}$. Television, as the main traditional media in China, is an important producer and disseminator of the public opinions to influence most Chinese people ${ }^{[9]}$. Whenever an emergency occurs, TV is the main platform, through which people may get an understanding of the development of such event. Song Jinbao and Chai Jianping analyzed and illustrated the radio and television public opinions from contents and methods researches on the air and the ratings, which is a systematic theory and practical results of study on the television public opinions ${ }^{[10]}$. Viewing data brand products of Gehua is also the representative product of television public opinion study ${ }^{[11]}$.

Firstly, we propose a scheme of cross-platform public opinion research and introduce the data layer, method layer and application layer. Next, we demonstrate the feasibility and practicability of the scheme with a practical example aiming at presenting the law and the essence of the public opinion propagation mode in a clear way, and excavating the relationship of propagation of public opinions among different media.

\section{Scheme of the Cross-platform Public Opinion Analysis}

The analysis scheme of cross-platform public opinions, as shown in Fig. 1, consists of the data layer, the method layer and the application layer.

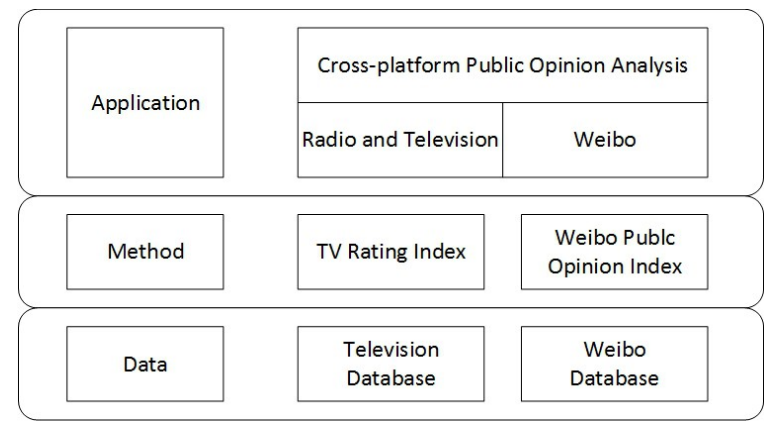

Figure 1: Chart of Cross-platform Public Opinion Analysis Scheme

Data layer: it includes television database and weibo database. The television database includes the program database and STB(Set Top Box) database from the Province Area Network(PAN) . The table of viewing records in STB database contains the user ID, the channel ID, the starting time for viewing and the ending time for viewing. Collected by a specific orientation network information acquisition system, weibo data includes all kinds of data related to hot event on weibo platform. We searched the amount of data on weibo through certain 
keyword like 'MH370' and different search conditions like "origin", "verify" and "time range", then we stored the result in weibo database. The data covers the amount of weibo, original weibo, verified weibo, and weibo geographical distribution data and weibo original content. etc. Some data is shown in Table 1:

\begin{tabular}{|c|l|l|c|c|}
\hline Date & Event name & Weibo post & Original weibo post & Verified weibo post \\
\hline $2014-03-08$ & the MH370 event & 785622 & 23713 & 47426 \\
\hline $2014-03-09$ & the MH370 event & 561895 & 43302 & 21651 \\
\hline$\ldots \ldots$ & $\ldots \ldots$ & $\cdots .$. & $\ldots$. & $\ldots$. \\
\hline
\end{tabular}

Table 1: Weibo Data

Method layer: it includes the calculation of TV rating index and weibo public opinion index.

\section{(1) Calculation of TV rating index:}

\section{Stage 1: Data cleansing}

Firstly, bond the viewing records for consecutive two days. The STB files save data day by day, the news programs may broadcast news at night and cross two days and a single record might be split into two. Next, remove invalid viewing records of which viewing time is less than one minute; finally, remove the viewing records that the audience remain watching the same channel for more than 5 hours before they watch the target news. In general, people's attention is limited to concentrate on the same channel for a very long time.

Stage 2: find out the broadcasting time list of the news related to the hot event

The event is usually broadcasted with the TV news program. If the content of news is all about the target event, the rating index of the event is the same to the news program; otherwise, we should find out the channel and time where and when the target event is broadcasted, and then prepare a broadcasting time list.

Stage 3: calculate the TV rating index

The rating indexes that we usually use to analyze the viewing results of the TV program include length of broadcast time, total viewing time, audience rating, arrival rate, fidelity, net inflow and market share. In this paper, we aim at calculating the rating index of hot event per day, so we regard different channels and different time during a certain day as the same.

Audience rating: it refers to the percentage of total viewing length of audiences watching a certain channel or program in viewing length of total audiences during a certain period. Get the rating index according to Formula (2.1).

$$
\text { Audience rating }=\frac{\sum_{\mathrm{i}=1}^{\mathrm{n}} \text { viewing } 1 \text { ength }_{\text {time } \mathrm{i}}}{\text { total viewing length } * \text { total audience }} * 100 \%
$$

Arrival rate: it refers to the percentage of the non-repetitive audience watching certain channel more than one minute for a certain time. The arrival rate can be obtained according to Formula (2.2).

$$
\text { Arrival rate }=\frac{\sum_{\mathrm{i}=1}^{\mathrm{n}} \text { Channel } \text { contact }_{\text {time } \mathrm{i}}}{\text { total audience }} * 100
$$

The audience rating takes the amount of audience and each person's viewing time into consideration, while the arrival rate just considers the amount of audience; therefore, the arrival rate just concerns about whether the audience contacts with the program during certain time but neglects the audience's viewing time.

Fidelity: the ratio of audience rating and arrival rate, range from $0 \%$ to $100 \%$. The higher the figure is, the more audience the program will attract. The fidelity can be calculated by Formula (2.3). 


$$
\text { Fidelity }=\frac{\text { audience } \text { rating }_{\text {channel }}}{\text { arriving } \text { rate }_{\text {channel }}} * 100
$$

The arrival rating and the audience's fidelity can reflect two dimensions of the evaluation of a channel or a program. The arrival rating can reflect the audience's scale and scope of that channel or program, namely, how many different audiences "contact" with that channel; and the fidelity demonstrates the depth of the TV rating rating, that is to say, the number of audiences who watch this channel or program from the beginning to the end.

Market share: it refers to the percentage of the audience viewing a certain channel or program in total audience watching TV during a certain time. The market share is calculated according to Formula (2.4).

$$
\text { Market share }=\frac{\text { audience } \text { rating }_{\text {channel }}}{\text { audience rating }} \text { all channel }^{\text {audien }}
$$

\section{(2) Calculation of weibo public opinion index}

Calculate the weibo indexes per day based on weibo database, including the number of weibo post, the number and the proportion of original weibo, the number and the proportion of the reposted weibo, the number and the proportion of the verified weibo. The amount of weibo post show the people's interest in the event, the number and the proportion of original weibo show interests of people who take the initiative and take action to learn about the latest news of certain event. The number and the proportion of the verified weibo show interests of people who has extensive influence and important leadership. The number and the proportion of the reposted weibo show interests of people who keeps a watchful eye on the event through others, reflecting the feedback of leadership of opinion leaders indirectly.

The application layer: it includes single-platform public opinion analysis and crossplatform public opinion analysis. By analyzing the variation tendency of index of the singleplatform public opinions in combination with the weibo content, we may find the factors that influence the propagation law of public opinion; moreover, we can analyze how public opinions on different platforms affect each other by comparing the tendencies of public opinions on different platforms.

\section{Case Analysis}

\subsection{Weibo Public Opinion Analysis}

This article takes the lost event of MH370 as an example to analyze the development of public opinions. The data collected by a directed network information system was about the discussion in terms of such event on weibo and used to calculate the weibo public opinion index per day. Some indexes are shown in Fig. 2.

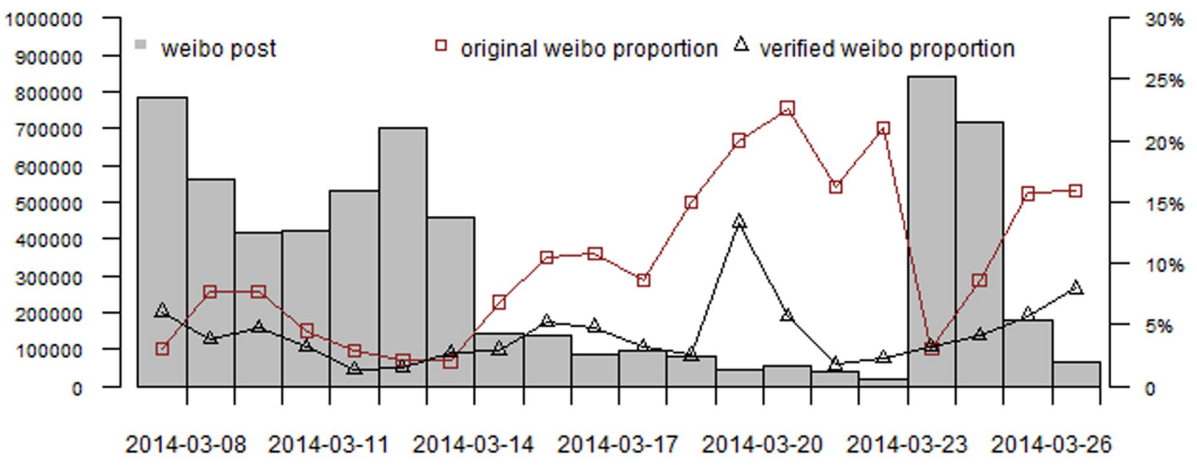

Figure 2: The Chart of the Tendency of Weibo Public Opinions 
In the morning of March 8, Malaysia airlines confirmed that MH370 lost contact with the control tower. At the beginning, the proportion of the original weibo was low while that of the verified weibo was very high. And a large number of reposted weibo suggested that verified users such as media and weibo masters have played an important role as the opinion leaders at the beginning; and a mass of ordinary users paid attention to it and repost the messages, which thus made the propagation quantity of the event soared on weibo. On second day, Malaysia airlines held a press conference in Beijing to inform people of the latest situation about MH370. At this moment, the netizens tended to be concerned about the whereabouts of the plane and the security of the passengers and crew, and prayed for the lost people, so the proportion of original weibo was promoted significantly. After that, as the media continued to follow up the latest news of rescue, the concerns in weibo were not so much as before. Until March 23, when the rescue was still going on but made no breakthrough, concerns about the event were decreasing; but there were still a lot of speculations and queries for this tragedy which caused the proportion of original weibo and verified users' weibo balloon. On March 24, Malaysia claimed that MH370 had crashed in the southern Indian Ocean. The extinction of hopes, the doubt of the Malaysian government, the pain and condolences to the victims, all about that made the quantity of weibo post reach a maximum, indicating the event had developed to the climax. Then the attention was plummeted and disappeared gradually for lack of new stimulus.

In conclusion, at the beginning of the emergency, the opinions of the authority of the news media and the verified users grabbed the public attention and detonated the topic, which resulted in a mass of discussion. Then all social parts responded to the event. With times went by, the public attention was declined and the event was not as white-hot as before, but there were still many users keeping on watching the development of the event and speculating the cause, demonstrating personal opinions and attitudes, so the proportion of original weibo rose. If there isn't significant breakthrough in the future, popular interest in the event is on the decline and people will no longer pay attention to the event.

\subsection{TV Public Opinion Analysis}

We collected 4590 videos about the lost event of MH370 from CNTV, and then removed the videos less than ten minutes, which cannot reflect the people's specific attitude to the event. Finally, 90 videos were left. We checked these videos and recorded the channel, date, actual viewing start time and end time of the lost event of MH370 as broadcasted in news program. Check the program list and make sure the videos information the same with the program list. Remove the record with duration less than 10 minutes. The final broadcasting time list shown in table 2 was used to calculate the viewing index.

\begin{tabular}{|l|l|l|c|}
\hline Channel ID & Jmname & Viewing Starting Time & Viewing Ending Time \\
\hline 00001 & The special report about the MH370 event & $2014 / 3 / 816: 00$ & $2014 / 3 / 817: 15$ \\
\hline 00002 & The special report about the MH370 event & $2014 / 3 / 911: 30$ & $2014 / 3 / 912: 00$ \\
\hline$\cdots \cdots$ & $\cdots \cdots$ & $\cdots \cdots$ & $\cdots \cdots$ \\
\hline
\end{tabular}

Table 2: Broadcasting Time List of the Event

According to the STB database and the broadcasting time list extracted from the program database and search results of CNTV, we calculate the viewing index. The viewing records from March 8, 2014 to March 25, 2014 in STB database take up more than 4GB of storage, including more than 80 million records, about 4.4 million per day. It takes about 5 minutes to calculate the 
rating indexes for one day when we take all channels broadcasting news about the event into consideration. In order to reduce the computational complexity but not affect viewing index evaluation results drastically, we only extract the viewing records whose channel is CCTV-X as the source of the main news reports at home and abroad and reduce the computation time to 3 minutes. The audience rating of famous news programs doesn't exceed $20 \%$, the arriving rate doesn't exceed $30 \%$ and the fidelity doesn't exceed $50 \%$ generally according to official data. The rating indexes we calculated is shown as Fig. 3.

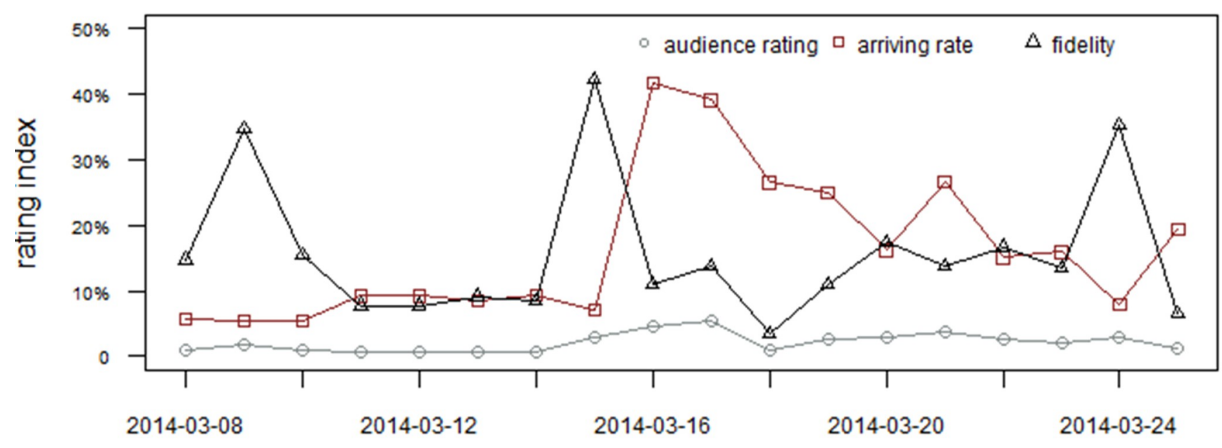

Figure 3: The Chart of the Tendency of Viewing Index

As shown in Fig. 3, upon occurrence of the lost event of MH370 on March 8, CCTV broadcasted special reports on it for half a month. On March 9, the first day the incident occurred, people paid highly attention to the event and the concerns reached a small peak. In terms of the viewing rating and fidelity, although a small crowd of people watched news, most of them watched the special reports for a long time. On March 15, the viewing rating and the arrival rate reached the maximum. During the next four days, the government paid much attention to the event and the TV media continued to report the latest news of the MH370 speculating and demonstrating the reason and the result of the lost plane. During this time, both the total broadcasting time of news and the total viewing time were the longest, and the viewing rating and the arrival rating remained high for a period of time, indicating that the audience cover the most range and the development of MH370 event on TV reached the climax. Later, attention of the event developed from the highs to lows, in spite of the falling of the viewing ratings and the arrival ratings, the loyalty was increasing because there were some loyal viewers still paying attention to the event on TV and nearly watching the whole reports.

\subsection{Analysis of Cross-platform Public Opinion}

Some public opinion index of weibo and TV platform are shown in Fig. 4.The audience rating reflects the viewing result about the MH370 event while the amount of the weibo post shows people's interest in the event; thus the comparison of the two indexes in the same chart can reflect the connected effects between the two platforms. As shown in Fig. 4, on March 8, when the lost event ofthe MH370 just occurred, the effect of the information communication on weibo was obvious, indicating that weibo would be more sensitive to emergency and react more quickly than TV media. On March 9, the authoritative news channels followed up and reported the latest news of the event. The rising of the viewing ratings and the falling down of the amount of weibo post indicated that the TV media controlled the situation quickly, making the discussions on the weibo to decline with the year-on-year growth of $28 \%$, as shown in Fig. 5 although it fell only a little. In a similar way, from March 14 to 16, the government made response to the speculations and queries spread on the internet by asking for experts to explain, which indicated the government paid enough attention to the event, consequently making the public calm down rapidly and making the discussions on the weibo to decline with year-on-year growth of 30\%-60\%. We can see that the weibo post and audience rating trade off and take turns from Fig. 5; therefore, it can be assumed that TV media and weibo interact each other in the 
propagation process of the emergent event. The report on TV can balance the heated debate on weibo and weibo also can exert important influence on the viewing effect of TV.

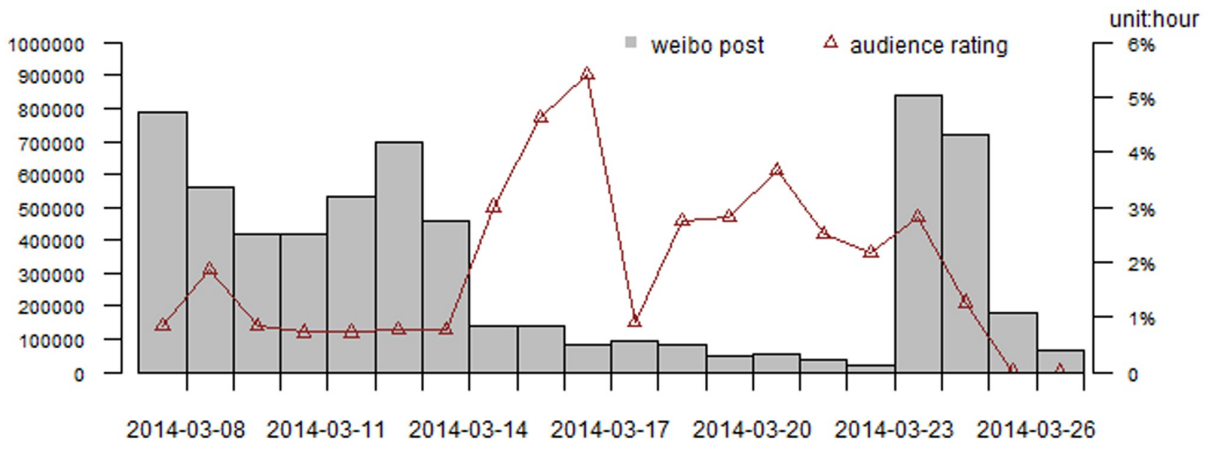

Figure 4: The Chart about the Tendency of Weibo Post and the Audience Rating

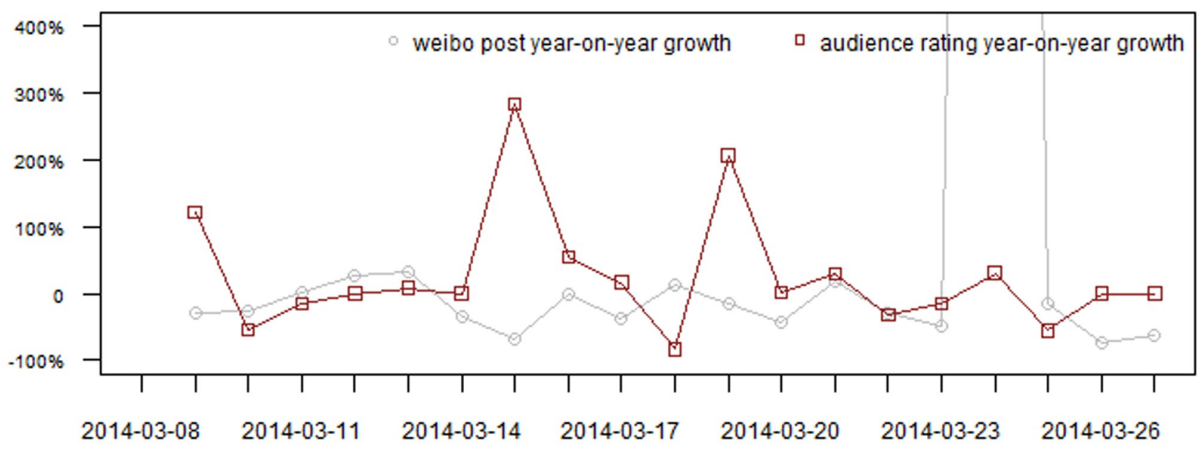

Figure 5: The Chart about Year-on-year Growth of Weibo Post and Year-on-year Growth of Audience Rating

\section{Conclusion}

In this paper, the MH370 event was taken as the example to show the tendency of public opinions, reasons and propagation rule about emergencies on weibo and TV platform. We calculate different indexes in respect of public opinions on different platforms with lower computational complexity and prepare charts of the public opinion on the single-platform and the cross-platform, which can reflect the changing tendency of the public opinions at different stages of development directly and effectively in different media. Through contrasting different indexes and the year-on-year growth of different indexes, we conclude that TV platform and weibo platform do have a linkage effect. The report on TV can balance the heated debate on weibo and weibo also can exert an important influence on the viewing effect of TV. The results also show that public opinions develop by following different laws in different media. In addition, the internet media response more quickly to the hot event compared with television media; therefore, the government may take different measures to control the development tendency of the hot event on different platforms.

\section{References}

[1] J.W. Zhu, The survival of media network in the digital media era[J].A news buffs(theoretic version).2008 (08):96-97 (In Chinese) 
[2] X.Q. Liu, C. Yin, Research about the domestic "media covergence"-based on the analysis of the almost 10 years serelevant literature published in "China journal full-text database" [J]. Zhaozhuang college newspaper.2008,25 (03):91-93 (In Chinese)

[3] H.B. Zhang, The researching about the public opinion of colleges and universities in Baidu Post Bar. [J].A news buff.2009(9):122-123 (In Chinese)

[4] W. Kang, The key point recognition of the emergency Internet public opinion based on SNS — taking the"7.23" event as an example[J]. Journal of public management. 2012, 9(3):101-111 (In Chinese)

[5] T.K. Das, D.P. Acharjya, M.R. Patra, Opinion mining about a product by analyzing public tweets in Twitter [C]. Proceedings of Computer Communication and Informatics(ICCCI),Coimbatore, INDIA, IEEE Press,2014:1- 4

[6] J.H. Ruan and W.X. Zhang, An efficient Spectral Algorithm for Network Community Discovery and Its Applications to Biological and Social Networks[C]. Proceedings of the 2007 Seventh IEEE International Conference on Data Mining, Omaha, NE, USA : IEEE Press, 2007:643-648.

[7] O. Amayri. N. Bouguila, Online news topic detection and tracking via localized feature selection[C]. Proceedings of Neural Networks (IJCNN), Dallas, TX, USA :IEEE Press, 2013:1-8

[8] W. Zhang, M.S. He, Influence of opinion leaders on dynamics and diffusion of network public opinion[C]. Proceedings of the 2013 International Conference on Management Science and Engineering (ICMSE), Harbin, P.R.China :IEEE Press, 2013: 139-144

[9] Z.B. Hong, Theory of how radio and television play the leading role of public opinion [J]. News Universe. 2011,(5):111-112 (In Chinese)

[10] J.B. Song, J.P. Chai. Radio and television public opinion analysis [J]. Journal of Communication University of China (natural science edition).2009,16(2):48-53 (In Chinese)

[11] Fusion network. Gehua launched official ratings brand "Gehua Release". [EB/OL] (2015-04-21). http://www.dwrh.net/a/gdw/yx/2014/1114/183670.html. (In Chinese)

[12] CSM Media Research. InfoSys terminology [ EB/OL ] $(2015-04-23)$. http://www.csm.com.cn/index.php/Knowledge/jargon. (In Chinese) 